\title{
MyoRing Implantation with and without Corneal Collagen Crosslinking for the Management of Keratoconus
}

\author{
Mehrdad Mohammadpour, MD; Ahmad Masoumi, MD; Mahmoud Dehghan, MD \\ Mohammad Nasser Hashemian, MD; Shahab Addin Karami, MD \\ Alireza Mahmoudi, MD
}

Translational Ophthalmology Research Center, Farabi Eye Hospital, Tehran University of Medical Sciences, Tehran, Iran

ORCID:

Mehrdad Mohammadpour: https://orcid.org/0000-0002-9383-6362

Ahmad Masoumi: https://orcid.org/0000-0002-9383-6362

\section{Abstract}

Purpose: To evaluate the safety and efficacy of femtosecond laser-assisted MyoRing implantation with concurrent corneal collagen crosslinking $(\mathrm{CXL})$ compared to MyoRing alone for the treatment of progressive keratoconus.

Methods: A total of 60 patients were enrolled in this randomized controlled trial. The patients were randomly allocated into two groups. In the first group, MyoRing was implanted, while in the second, it was inserted in the corneal stroma using the same technique, along with simultaneous CXL. Visual, refractive, topographic, and abberometric outcomes were measured preoperatively and at every postoperative visit.

Results: Data of 47 patients were available at the end of the study; 28 in the MyoRing group and 19 in the MyoRing + CXL group. The mean uncorrected distance visual acuity (UDVA) improved from $0.79 \pm 0.39 \log M A R$ to $0.52 \pm 0.31 \operatorname{logMAR}(P<0.05)$ in the MyoRing $+C X L$ group and from $0.65 \pm 0.38 \log M A R$ to $0.62 \pm 0.23 \log M A R(P=0.70)$ in the MyoRing group. CDVA changed from $0.33 \pm 0.19 \log M A R$ to $0.25 \pm 0.16 \log M A R(P=0.10)$ in the MyoRing $+C X L$ group and 0.32 $\pm 0.22 \log M A R$ to $0.33 \pm 0.17 \log M A R(P>0.50)$ in the MyoRing group. The mean keratometry $(\mathrm{Km})$ decreased from $47.5 \pm 2.7 \mathrm{D}$ to $43.8 \pm 3.2 \mathrm{D}(\mathrm{P}<0.001)$ in the MyoRing group and 49.3 $\pm 3.4 \mathrm{D}$ to $45.1 \pm 3.0 \mathrm{D}(\mathrm{P}<0.001)$ in the MyoRing $+\mathrm{CXL}$ group. Besides, horizontal coma was significantly lower in the MyoRing + CXL group $(P=0.022)$.

Conclusion: MyoRing insertion combined with $\mathrm{CXL}$ is a safe and effective method for the treatment of keratoconus. The visual and topographic outcomes were comparable to that for MyoRing insertion after 10 months; however, horizontal coma was significantly lower in the MyoRing + CXL group.

Keywords: Corneal Collagen Crosslinking; Keratoconus; MyoRing

J Ophthalmic Vis Res 2020; 15 (4): 486-492

Correspondence to:

Ahmad Masoumi, MD. Translational Ophthalmology Research Center, Farabi Eye Hospital, Tehran University of Medical Sciences, Qazvin Sq, Tehran 13366, Iran. E-mail: ahmad.masoomi1990@gmail.com

Received: 21-05-2019 Accepted: 25-06-2020

\section{Access this article online}

Website:

https://knepublishing.com/index.php/JOVR

DOI:

10.18502/jovr.v15i4.7790

\section{INTRODUCTION}

Keratoconus is a non-inflammatory corneal ectasia resulting in progressive thinning and steepening

This is an open access journal, and articles are distributed under the terms of the Creative Commons Attribution-NonCommercial-ShareAlike 4.0 License, which allows others to remix, tweak, and build upon the work non-commercially, as long as appropriate credit is given and the new creations are licensed under the identical terms.

How to cite this article: Mohammadpour M, Masoumi $A$, Dehghan $M$, Hashemian MN, Karami SA, Mahmoudi A. MyoRing Implantation with and without Corneal Collagen Crosslinking for the Management of Keratoconus. J Ophthalmic Vis Res 2020;15:486-492. 
of the cornea. Patients usually present with decreased visual acuity, progressive myopia, irregular astigmatism, and central corneal scarring. ${ }^{[1]}$

A wide range of options is available for the treatment of keratoconus. In early stages, patients can be treated with spectacles or contact lenses. However, in advanced stages with corneal scarring, corneal transplantation is the only available option to restore vision. For patients without corneal scarring intolerant to contact lenses, the new modality, intracorneal ring segment (ICRS) or continuous intracorneal ring implantation in the corneal stroma may be used. The technique of insertion of a continuous intracorneal ring was developed in the late $20^{\text {th }}$ century with the aim of correcting myopia. However, owing to technical difficulties and incision-related complications, continuous intracorneal rings were supplanted by ICRS, which had been used to correct mild to moderate myopia, ${ }^{[2-4]}$ but recently gained great popularity to treat keratoconus. ${ }^{[5-7]}$ Although ICRS cannot completely avert corneal transplantation, it can delay the keratoconus progression. ${ }^{[8]}$ ICRSs can flatten the corneal center and move the corneal apex to the center of cornea. It facilitates the fitting of the contact lens and optimizes the best corrected visual acuity.

The introduction of femtosecond laser technology in the field of refractive surgery offered new hopes to treat human refractive errors and also provided a new surgical modality to create tunnels for precise ICRS insertion. The femtosecond laser-assisted ICRS implantation has several advantages over mechanical approaches including a more uniform dissection, less patient discomfort, quick recovery, and more predictable results. ${ }^{[9]}$

Corneal collagen crosslinking (CXL) uses ultraviolet A (UVA) light and riboflavin to stiffen the corneal stroma. The irradiation of riboflavin results in formation of free radicals, inducing the formation of covalent bonds between the amino acid groups of collagen fibers. ${ }^{[10,11]}$ It halts the progression of keratoconus as shown by several studies. ${ }^{[10-13]}$ ICRS insertion can improve the corneal topography and correct myopia and astigmatism in keratoconic corneas; however, it has limited effect on the progression of keratoconus. In comparison, as stated earlier, CXL is a safe and effective method to stop the progression of ectatic corneal disorders. Thus, hypothetically, the benefits of both methods can be obtained by combining the two procedures. Several studies have been performed to test the safety and efficacy of the combined procedure. ${ }^{[14-17]}$ However, only few studies have evaluated same-day ICRS + CXL. This study was designed to assess and compare the long-term outcome of simultaneous insertion of MyoRing and CXL with MyoRing alone.

\section{METHODS}

This study was approved by the Institutional Review Board of Tehran University of Medical Sciences and was compliant with the tenets of the Declaration of Helsinki. Informed consent was obtained from all patients prior to the study. A total of 60 patients aged between 18 and 35 years who were diagnosed with keratoconus based on the clinical and Pentacam criteria were enrolled in this study. The inclusion criteria of the study were keratoconic eyes with central clear cornea, contact lens intolerance, and central corneal thickness $>380 \mu \mathrm{m}$. Patients who had previously undergone any ophthalmic surgery, pregnant and lactating women, and those with a history of collagen vascular diseases were excluded from this study. Patients were randomly allocated into two groups. In the first group, MyoRing was implanted at a depth of $300 \mu \mathrm{m}$ using femtosecond laser. In the second group, MyoRing was implanted using the same technique with simultaneous CXL. Preoperative and postoperative examinations included uncorrected distance visual acuity (UDVA), corrected distance visual acuity (CDVA), dry and cycloplegic refraction, slit lamp biomicroscopy, dilated fundus examination, topographic and optical pachymetry using the Pentacam system (OCULUS, Wetzlar, Germany), and corneal abbermotry (iTrace, Tracey technologies, Houston, TX). Patients were examined one day before the surgery and one day, one month, three months, and ten months postoperatively. The information for this trial is available to the public through the Iranian National Registry for Clinical Trials (http://www.irct.ir).

\section{Surgical technique}

All surgeries were done by the same experienced surgeon (MM) at Farabi Eye Hospital, Tehran, 
Iran. The procedure was performed under topical anesthesia using tetracaine $0.5 \%$ eye drops. The eyes were sterilized using povidone iodine, and an eyelid speculum was used to hold the eyes open. The cornea was marked in the pupillary center by a Sinskey hook as a reference point for pocket creation. A $300 \mu \mathrm{m}$ deep tunnel was created in the corneal stroma using the femtosecond laser (Technolas 520F..........). The laser creates dissection planes by focusing a $3 \mu \mathrm{m}$ diameter laser beam with a frequency of $500 \mathrm{kHz}$ and a wavelength of $1054 \mathrm{~nm}$ at a predetermined stromal depth. A MyoRing with a diameter of $5 \mathrm{~mm}$ and a thickness of $240 \mu \mathrm{m}$ was chosen for implantation. It was then inserted into the corneal pocket via a previously made temporal corneal incision. In the second group, after anesthetizing the eye and creating the pocket, riboflavin $0.1 \%$ was injected into the corneal pocket to completely fill it. After $5 \mathrm{~min}$, diffusion of riboflavin was observed in the corneal stroma and anterior chamber. The MyoRing was then implanted using the mentioned technique. Finally, the cornea was exposed to 370-nm UVA light for $10 \mathrm{~min}$ with an irradiance of $9 \mathrm{~mW} / \mathrm{cm}^{2}$. The MyoRing implantation and CXL procedure was uneventful without needing sutures.

Topical antibiotic and steroid drops, and artificial tears were used postoperatively. Chloramphenicol $0.5 \%$ was used every 4 hours for the first 10 days. Betamethasone eye drops were prescribed for every 2 hours after the procedure and gradually tapered in the following three months.

\section{Statistical analysis}

Statistical analysis was performed using the SPSS, version 21 (SPSS Inc., Chicago, IL). A P-value < 0.05 was considered as significant. Normality of data was tested suing the Kolmogorov-Smirnov test. The Wilcoxon rank sum test was used to compare the postoperative and preoperative values. For comparison of outcomes between the two groups, the Mann-Whitney $U$ test was performed.

\section{RESULTS}

Data of 47 eyes of 47 patients were available at the end of the study; 28 patients in the MyoRing group and 19 in the MyoRing + CXL group. The two study groups were matched before treatment in terms of age, UDVA, CDVA, pachymetry, keratometry $(\mathrm{Km})$, and higher order aberrations. Table 1 shows the preoperative values.

\section{Visual Outcomes}

After 10 months, the mean UDVA improved from $0.79 \pm 0.39 \log$ MAR to $0.52 \pm 0.31 \log$ MAR $(P<$ $0.05)$ in the MyoRing + CXL group. However, such an improvement was not observed in the MyoRing group. The improvement in CDVA was statistically insignificant in both groups at the end of followup. The increase in UDVA was observed after six months in the MyoRing + CXL group.

\section{Refractive Outcomes}

Statistical analysis revealed that the spherical equivalent (SE) improved from $-6.51 \pm 3$ to $-1.80 \pm$ 2 in the MyoRing group $(P<0.001)$ after 10 months. In the MyoRing + CXL group, the SE improved from $-6.63 \pm 2.5$ to $-1.7 \pm 2(P<0.001)$. There was a significant improvement in Kmax in the MyoRing $+C X L$ group $(P<0.05)$. The mean $\mathrm{Km}$ improved significantly in both groups $(P<0.001)$.

\section{Corneal Aberrations}

The mean root mean square (RMS) total decreased by $0.55(P>0.1)$ in the MyoRing $+C X L$ group, while it increased by $0.95 \mu \mathrm{m}$ in the MyoRing group $(P>$ 0.05). The mean RMS of higher order aberrations (RMS HOA) increased by 0.01 in the MyoRing + CXL group $(P>0.1)$ and by $0.71 \mu \mathrm{m}$ in the MyoRing group $(P<0.05)$. We also observed that the horizontal coma decreased by $0.37 \mu \mathrm{m}$ in the MyoRing $+\mathrm{CXL}$ group $(P<0.05)$. Tables 2 and 3 show the visual and topographic outcomes in the two groups after 10 months.

\section{Comparison of MyoRing Insertion and MyoRing Insertion + CXL}

After 10 months, no significant difference was observed in the UDVA, CDVA, and Kmax in patients who underwent MyoRing insertion alone and those who underwent MyoRing + CXL. However, horizontal coma was significantly lower in the group that underwent MyoRing insertion associated with CXL. Table 4 compares the refractive and visual outcomes in both groups after 10 months. 
Table 1. Mean of the preoperative data

\begin{tabular}{lccc}
\hline & MyoRing group & MyoRing + CXL group & P-value \\
\hline Age & $26.1 \pm 4.6$ & $24.9 \pm 5.2$ & 0.360 \\
UDVA (logMAR) & $0.65 \pm 0.38$ & $0.79 \pm 0.39$ & 0.200 \\
CDVA (logMAR) & $0.32 \pm 0.22$ & $0.33 \pm 0.19$ & 0.878 \\
Spherical equivalent (D) & $-6.51 \pm 3.01$ & $-6.63 \pm 2.51$ & 0.745 \\
Km (D) & $47.5 \pm 2.7$ & $49.3 \pm 3.4$ & 0.055 \\
K max (D) & $52.4 \pm 5.19$ & $54.2 \pm 4.7$ & 0.221 \\
Thinnest point $(\mu \mathrm{m})$ & $449 \pm 41$ & $441 \pm 32$ & 0.551 \\
Coma horizontal $(\mu \mathrm{m})$ & $0.70 \pm 0.65$ & $0.73 \pm 0.46$ & 0.735 \\
Coma vertical $(\mu \mathrm{m})$ & $1.39 \pm 1.07$ & $1.19 \pm 1.05$ & 0.231 \\
RMS HOA $(\mu \mathrm{m})$ & $2.19 \pm 1.31$ & $2.05 \pm 0.74$ & 0.734
\end{tabular}

UDVA, uncorrected distance visual acuity; CDVA, corrected distance visual acuity; logMAR, logarithm of minimum of angle of resolution; Km, mean keratometry; RMS HOA, root mean square value of total higher order aberrations in a simulated 6 mm pupil

Table 2. Change in variables in the MyoRing group after 10 months of follow-up compared to baseline

\begin{tabular}{lccc}
\hline & Preoperative data & Postoperative 10- month data & P-value \\
\hline UDVA (logMAR) & $0.65 \pm 0.38$ & $0.62 \pm 0.23$ & 0.710 \\
CDVA (logMAR) & $0.32 \pm 0.22$ & $0.33 \pm 0.17$ & 0.906 \\
Spherical equivalent $(\mathrm{D})$ & $-6.51 \pm 3.07$ & $-1.8 \pm 2.11$ & 0.001 \\
K max $(\mathrm{D})$ & $52.4 \pm 5.19$ & $51.5 \pm 4.1$ & 0.312 \\
Thinnest point $(\mu \mathrm{m})$ & $449 \pm 41$ & $452 \pm 40$ & 0.423 \\
Coma horizontal $(\mu \mathrm{m})$ & $0.70 \pm 0.65$ & $0.99 \pm 0.6$ & 0.123 \\
Coma vertical $(\mu \mathrm{m})$ & $1.39 \pm 1.08$ & $2.01 \pm 1.11$ & 0.021 \\
RMS HOA $(\mu \mathrm{m})$ & $2.19 \pm 1.31$ & $2.91 \pm 1.23$ & 0.006
\end{tabular}

\section{Complications}

No complications such as migration of the MyoRing into the anterior chamber were observed during the surgery. As stated earlier, the procedure was uneventful in all of the eyes, and sutures were not needed. All eyes showed excellent tolerance to the implanted MyoRings, and no migration or extrusion was observed. Moreover, none of the eyes developed corneal ulcers or stromal necrosis superficial to the segment.

\section{DISCUSSION}

This study compared the refractive and corneal aberrometric effect of MyoRing insertion alone and MyoRing with concurrent CXL. As shown in the results, combining $\mathrm{CXL}$ with MyoRing implantation is an efficient method for keratoconus treatment and can significantly decrease the $\mathrm{Km}$ values. It can significantly increase the UDVA and decrease the SE. When compared to the MyoRing group, horizontal coma was significantly lower in the MyoRing + CXL group; however, there was no significant difference in other topographic and refractive outcomes.

The main indication of $\mathrm{CXL}$ is to halt the progression of ectatic corneal disorders such as keratoconus, pellucid marginal degeneration, and post-laser in-situ keratomileusis (LASIK) corneal ectasia. ${ }^{[14,18,19]}$ Collagen fibrils are crucial for corneal stability. It has been demonstrated that the diagonal links between collagen fibrils are significantly reduced in keratoconic corneas. ${ }^{[20]}$ This leads to corneal thinning in central and paracentral areas and causes myopia, irregular astigmatism, and decreased visual acuity. CXL stabilizes the diseased cornea by creating covalent 
Table 3. Change in variables in the MyoRing + CXL group after 10 months of follow-up compared to baseline

\begin{tabular}{|c|c|c|c|}
\hline & Preoperative data & Postoperative 10- month data & P-value \\
\hline UDVA (logMAR) & $0.79 \pm 0.39$ & $0.52 \pm 0.31$ & 0.034 \\
\hline CDVA (logMAR) & $0.33 \pm 0.19$ & $0.25 \pm 0.16$ & 0.118 \\
\hline Spherical equivalent (D) & $-6.63 \pm 2.51$ & $-1.70 \pm 2.41$ & 0.001 \\
\hline $\mathrm{K} \max (\mathrm{D})$ & $54.2 \pm 4.71$ & $52.1 \pm 3.75$ & 0.032 \\
\hline Thinnest point $(\mu \mathrm{m})$ & $442 \pm 39$ & $445 \pm 51$ & 0.623 \\
\hline Coma horizontal $(\mu \mathrm{m})$ & $0.73 \pm 0.46$ & $0.37 \pm 0.31$ & 0.033 \\
\hline Coma vertical $(\mu \mathrm{m})$ & $1.91 \pm 1.14$ & $1.62 \pm 1.42$ & 0.081 \\
\hline RMS HOA $(\mu \mathrm{m})$ & $2.05 \pm 0.74$ & $2.06 \pm 0.18$ & 0.878 \\
\hline \multicolumn{4}{|c|}{ Table 4. Comparison of parameters in the MyoRing and MyoRing $+C X L$ groups after 10 months } \\
\hline & MyoRing & MyoRing + CXL & P-value \\
\hline UDVA (logMAR) & $0.62 \pm 0.23$ & $0.52 \pm 0.31$ & 0.174 \\
\hline CDVA (logMAR) & $0.33 \pm 0.17$ & $0.25 \pm 0.16$ & 0.105 \\
\hline Spherical equivalent (D) & $-1.8 \pm 2.11$ & $-1.70 \pm 2.41$ & 0.920 \\
\hline $\mathrm{Km}(\mathrm{D})$ & $43.8 \pm 3.2$ & $45.1 \pm 3.0$ & 0.137 \\
\hline $\mathrm{K} \max (\mathrm{D})$ & $51.5 \pm 4.1$ & $52.1 \pm 3.75$ & 0.346 \\
\hline Thinnest point $(\mu \mathrm{m})$ & $452 \pm 40$ & $445 \pm 51$ & 0.688 \\
\hline Coma horizontal $(\mu \mathrm{m})$ & $0.99 \pm 0.6$ & $0.37 \pm 0.31$ & 0.022 \\
\hline Coma vertical $(\mu \mathrm{m})$ & $2.01 \pm 1.11$ & $1.62 \pm 1.42$ & 0.661 \\
\hline RMS HOA $(\mu \mathrm{m})$ & $2.91 \pm 1.23$ & $2.06 \pm 0.18$ & 0.896 \\
\hline
\end{tabular}

bonds (cross links) between the collagen fibrils. It stops the progression of keratoconus without considerably changing the shape of the cornea. MyoRing implantation, on the other hand, can treat keratoconus by inducing a flattening effect, without affecting the underlying pathophysiology of ectasia. Therefore, combining CXL with MyoRing implantation would hypothetically stabilize the progression of keratoconus and improve the visual acuity by flattening the cornea. There are limited studies evaluating the outcome of MyoRing implantation with simultaneous $\mathrm{CXL}$.

$\mathrm{CXL}$, which has been used for treating keratoconus since 1990s, is believed to be the only treatment that can halt the progression of keratoconus. In this procedure, UVA light is irradiated to the cornea after treatment with riboflavin solution, resulting in formation of free radicals and inducing covalent bonds between amino groups of the collagen molecule, thus increasing the biomechanical stability of the cornea. $^{[21,22]}$ In this study, we used a rather new technique for $\mathrm{CXL}$; riboflavin $0.1 \%$ solution was injected into the corneal pocket created by the femtosecond laser, followed by exposing the cornea to UVA light for $10 \mathrm{~min}$. The corneal epithelium is usually removed before the UV light is irradiated to the cornea to allow adequate penetration of riboflavin into the corneal stroma. Simultaneous introduction of riboflavin $0.1 \%$ into the corneal pocket created by the femtosecond laser obviates the need for epithelial debridement and reduces pain and discomfort in the early postoperative period. ${ }^{[23,24]}$ However, the corneal stroma composed of tightly compacted collagen fibers is fairly resistant to molecular transport. The stromal barrier can be eliminated by injecting the riboflavin solution directly into the pocket, allowing more uniform distribution in the corneal stroma. Dextran was not present in the solution as corneal toxicity might occur when it is directly injected into the corneal stroma. It has been demonstrated that a cross-linked cornea is less clear compared to a virgin cornea. Therefore, femtosecond laser 
penetration into the corneal stroma may be less effective in eyes that underwent CXL. El Raggal found that channel creation by femtosecond laser is more challenging in eyes that had undergone $\mathrm{CXL}$. They attributed this effect to the possible rigidity of collagen fibers in the deep corneal stroma in patients treated by CXL. ${ }^{[25]}$ Femtosecond laser energy can be increased to overcome this problem; however, this solution may lead to a persistent corneal reaction, postoperatively. ${ }^{[25]}$

The keratoconic cornea is a highly aberrated cornea with significantly more coma and comalike aberrations compared to the normal eye. In this study, horizontal coma was significantly lower in eyes that underwent MyoRing implantation with simultaneous CXL. Thus, combining CXL with MyoRing implantation might enhance the flattening effect of the segment without inducing considerable HOA. Furthermore, a synergistic effect might also be present, when CXL is combined with ICRS implantation, augmenting the therapeutic effect that is observed with either treatment. ${ }^{[15]}$

Hafez recently studied the outcomes of KeraRing and MyoRing implantation with simultaneous CXL. ${ }^{[15]}$ Patients who underwent MyoRing implantation plus CXL showed a significant reduction in Kmean. However, there was little improvement in the astigmatic component of keratoconus with MyoRing implantation, and significant reduction of astigmatism was observed in patients treated by combination of KeraRing and CXL.

In a retrospective study by Bikbova et al, the efficacy of MyoRing implantation and MyoRing implantation + CXL after three years was reported. They found that MyoRing implantation with simultaneous CXL had slightly better outcomes, although the effect of MyoRing implantation alone was stable over time. ${ }^{[26]}$ Similarly, in this study, we found that the topographic and visual outcomes were comparable in the two groups after 10 months, although horizontal coma was significantly lower in patients who underwent MyoRing implantation with simultaneous CXL.

El Raggal evaluated the outcome of combined KeraRing insertion with CXL, performed in a single session or with a six-month interval. He observed that there was a significant improvement in UDVA, CDVA, and keratometric values. However, patients treated using the same-day method had better topographic outcomes. ${ }^{[27]}$
Coskunseven and colleagues found that ICRS implantation followed by $\mathrm{CXL}$ is more effective in improving CDVA, SE, and the mean $\mathrm{Km}$ in keratoconus compared to CXL followed by ICRS implantation. The mean interval between the treatments was seven months. ${ }^{[28]}$

None of the patients during the study developed segment decentration and extrusion, partly due to application of femtosecond laser, which creates a more precise depth of incision.

Despite the limited number of cases, this study shows that the MyoRing $+C X L$ procedure is a safe and effective method for treating keratoconus. It is comparable to MyoRing insertion alone for improvement in visual and topographic outcomes in the short term. Moreover, a significant reduction in $\mathrm{HOA}$ is observed with the MyoRing $+\mathrm{CXL}$ procedure, which is of paramount importance in highly aberrant corneas with keratoconus. More studies with a longer follow-up are needed to better elucidate the efficacy and safety of this procedure.

\section{Financial Support and Sponsorship}

Nil.

\section{Conflicts of Interest}

There are no conflicts of interest.

\section{REFERENCES}

1. Rabinowitz YS. Keratoconus. Surv Ophthalmol 1998;42:297-319.

2. Assil KK, Barrett AM, Fouraker BD, Schanzlin DJ. One-year results of the intrastromal corneal ring in nonfunctional human eyes. Arch Ophthalmol 1995;113:159-167.

3. Nosé W, Neves RA, Burris TE, Schanzlin DJ, Belfort R. Intrastromal corneal ring: 12-month sighted myopic eyes. J Refract Surg 1996;12:20-28.

4. Schanzlin DJ, Asbell PA, Burris TE, Durrie DS. The Intrastromal Corneal Ring Segments: Phase II Results for the Correction of Myopia. Ophthalmology 1997;104:10671078.

5. Colin J, Cochener B, Savary G, Malet F. Correcting keratoconus with intracorneal rings. J Cataract Refract Surg 2000;26:1117-1122.

6. Colin J, Cochener B, Savary G, Malet F, Holmes-Higgin D. INTACS inserts for treating keratoconus: one-year results. Ophthalmology 2001;108:1409-1414.

7. Wachler BSB, Chandra NS, Chou B, Korn TS, Nepomuceno $\mathrm{R}$, Christie JP. Intacs for keratoconus. Ophthalmology 2003;110:1031-1040. 
8. Jabbarvand M, SalamatRad A, Hashemian H, Mazloumi M, Khodaparast M. Continuous intracorneal ring implantation for keratoconus using a femtosecond laser. J Cataract Refract Surg 2013;39:1081-1087.

9. Ratkay-Traub I, Ferincz IE, Juhasz T, Kurtz RM, Krueger RR. First clinical results with the femtosecond neodyniumglass laser in refractive surgery. $J$ Refract Surg 2003;19:94-99.

10. Raiskup F, Spoerl E. Corneal crosslinking with riboflavin and ultraviolet A. Part II. Clinical indications and results. Ocul Surf 2013;11:93-108.

11. Caporossi A, Baiocchi S, Mazzotta C, Traversi C, Caporossi T. Parasurgical therapy for keratoconus by riboflavinultraviolet type A rays induced cross-linking of corneal collagen: preliminary refractive results in an Italian study. $J$ Cataract Refract Surg 2006;32:837-845.

12. Caporossi A, Mazzotta C, Baiocchi S, Caporossi T. Longterm results of riboflavin ultraviolet a corneal collagen cross-linking for keratoconus in Italy: the Siena eye cross study. Am J Ophthalmol 2010;149:585-593.

13. Kymionis GD, Grentzelos MA, Liakopoulos DA Paraskevopoulos TA, Klados NE, Tsoulnaras KI, et al. Long-term follow-up of corneal collagen cross-linking for keratoconus-the Cretan study. Cornea 2014;33:10711079.

14. Hafezi F, Kanellopoulos J, Wiltfang R, Seiler T. Corneal collagen crosslinking with riboflavin and ultraviolet $A$ to treat induced keratectasia after laser in situ keratomileusis. J Cataract Refract Surg 2007;33:2035-2040.

15. Hafez Ml. Combined cross-linking with femtosecond laser Myoring implantation versus combined cross-linking with femtosecond laser Keraring implantation for treatment of keratoconus. Delta J Ophthalmol 2016;17:1-8.

16. Kazakbaeva G, Bikbov M, Usubov E. The evaluation of intrastromal MyoRing implantation with corneal collagen cross-linking in keratoconus treatment. Acta Ophthalmologica 2016;94:S256.

17. Wang SL, Kanellopoulos AJ. Safety and efficacy of crosslinking following intacs implantation for the stabilization of keratoconus. Invest Ophthalmol Vis Sci 2011;52:5200.
18. Wollensak G. Crosslinking treatment of progressive keratoconus: new hope. Curr Opin Ophthalmol 2006;17:356-360.

19. Spadea L. Corneal collagen cross-linking with riboflavin and UVA irradiation in pellucid marginal degeneration. $J$ Refract Surg 2010;26:375-377.

20. Sherwin T, Brookes $\mathrm{NH}$. Morphological changes in keratoconus: pathology or pathogenesis. Clin Experiment Ophthalmol 2004;32:211-217.

21. Gkika M, Labiris G, Kozobolis V. Corneal collagen crosslinking using riboflavin and ultraviolet-A irradiation: a review of clinical and experimental studies. Int Ophthalmol 2011;31:309-319.

22. Meiri Z, Keren S, Rosenblatt A, Sarig T, Shenhav L, Varssano D. Efficacy of corneal collagen cross-linking for the treatment of keratoconus: a systematic review and meta-analysis. Cornea 2016;35:417-428.

23. Leccisotti A, Islam T. Transepithelial corneal collagen cross-linking in keratoconus. J Refract Surg 2010;26:942948.

24. Kanellopoulos AJ. Collagen cross-linking in early keratoconus with riboflavin in a femtosecond lasercreated pocket: initial clinical results. J Refract Surg 2009;25:1034-1037.

25. El-Raggal TM. Effect of corneal collagen crosslinking on femtosecond laser channel creation for intrastromal corneal ring segment implantation in keratoconus. $J$ Cataract Refract Surg 2011;37:701-705.

26. Bikbova G, Kazakbaeva G, Bikbov M, Usubov E. Complete corneal ring (MyoRing) implantation versus MyoRing implantation combined with corneal collagen crosslinking for keratoconus: 3-year follow-up. Int Ophthalmol 2018;38:1285-1293.

27. El-Raggal TM. Sequential versus concurrent KERARINGS insertion and corneal collagen cross-linking for keratoconus. Br J Ophthalmol 2011;95:37-41.

28. Coskunseven E, Jankov MR, Hafezi F, Atun S, Arslan $E$, Kymionis GD. Effect of treatment sequence in combined intrastromal corneal rings and corneal collagen crosslinking for keratoconus. J Cataract Refract Surg 2009;35:2084-2091. 